\title{
Identification of REST targets in the Xenopus tropicalis genome
}

\author{
Banu Saritas-Yildirim ${ }^{1}$, Christopher P Childers ${ }^{1,2}$, Christine G Elsik ${ }^{1,2,3}$ and Elena M Silva ${ }^{1 *}$
}

\begin{abstract}
Background: A major role of REST (repressor element-1 silencing transcription factor) is to inhibit the expression of neuronal genes in neural stem cells and non-neuronal cells by binding to a 21 bp consensus sequence and recruiting epigenetic and regulatory cofactors to gene regulatory regions. In neural stem cells, REST silences differentiation-promoting genes to prevent their premature expression and is central to the regulation of neurogenesis and the balance of neural stem cells and neurons.

Results: To understand the role of REST in vertebrate neurogenesis, we performed a genome-wide screen for REST targets in Xenopus tropicalis. We identified 742 neuron-restrictive silencer elements (NRSE) associated with 1396 genes that are enriched in neuronal function. Comparative analyses revealed that characteristics of NRSE motifs in frog are similar to those in mammals in terms of the distance to target genes, frequency of motifs and the repertoire of putative target genes. In addition, we identified four F-box ubiquitin ligases as putative REST targets and determined that they are expressed in neuronal tissues during Xenopus development.
\end{abstract}

Conclusion: We identified a conserved core of putative target genes in human, mouse and frog that may be fundamental to REST function in vertebrates. We demonstrate that NRSE sites are associated with both protein-coding genes and IncRNAs in the human genome. Furthermore, we demonstrate that REST binding sites are abundant in low gene-occupancy regions of the human genome but this is not due to an increased association with non-coding RNAs. Our findings identify novel targets of REST and broaden the known mechanism of REST-mediated silencing in neurogenesis.

Keywords: REST, NRSE, Xenopus, Genome-wide screen, Neurogenesis, Gene silencing

\section{Background}

The repressor element-1 silencing transcription factor (REST, also called NRSF for neuron-restrictive silencer factor) silences neuronal genes in non-neuronal tissues and in neural stem cells of vertebrates to restrict their expression to neurons and to prevent premature differentiation, respectively [1,2]. REST binds to a conserved 21-bp neuron-restrictive silencing element (NRSE) in the flanking regulatory regions or introns of many neuronal genes $[3,4]$ and recruits the cofactors CoREST [5] and Sin3A [6] to form repressor complexes with histone deactylases [7], histone modifying proteins [8], the methyl-CpG-binding protein $\mathrm{MeCP}[9,10]$ and components of the SWI-SNF chromatin remodeling complexes [11]. Together these

\footnotetext{
* Correspondence: elena.silva@georgetown.edu

'Department of Biology, Georgetown University, 411 Regents Hall, Washington, DC 20057, USA

Full list of author information is available at the end of the article
}

proteins change the architecture of DNA to heterochromatin and silence target genes [9].

REST is critical for the maintenance of neural stem cells [12-15] and the regulation of neurogenesis. For neuron specific genes to be expressed and neurogenesis to proceed, REST activity is diminished in neural stem cells by two mechanisms; REST transcripts are down-regulated [16] and REST protein is targeted for degradation in the proteasome by the beta-tranducin repeat containing/F-box protein with WD40 domain 1 ( $\beta$-TRCP/Fbxw1 ubiquitin ligase) [17]. Although REST is most commonly reported to silence the expression of protein-coding genes, recent studies suggest that it also regulates non-coding RNAs involved in neurogenesis [18-21]. For example, the neuron specific microRNAs miR-9* and miR-124, important for repression of BAF53a (Brg/Brm association factor 53a) mediated chromatin remodeling and cell cycle exit, are repressed by REST in neural progenitors [20]. REST has 
also been shown to regulate and interact with long non-coding RNAs (lncRNAs) to control neurogenesis [21-24]. As an example, the nuclear lncRNA, lncRNA_N1, physically interacts with REST during differentiation of hESCs (human embryonic stem cells) to promote neurogenesis [23].

In this study, we performed a screen for REST binding sites and targets in the genome of the diploid amphibian Xenopus tropicalis, a model system for genetics and development. Using a degenerate 17 bp sequence derived from 32 bona fide REST targets, we identified 742 NRSE motifs associated with 1396 protein-coding genes. The NRSE distance to genes, number of NRSEs per gene, and the suite of neuronal genes associated with NRSEs in frog are conserved with mouse and human. Through a literature search, we identified which putative target genes are expressed in neuronal tissues and with expression analysis, we verified the restriction of expression of four F-box genes to neuronal tissues. In addition, we found that NRSEs are associated with long noncoding RNAs but not other classes of non-coding RNAs in the human genome.

\section{Results}

\section{Identification of NRSE sites in the Xenopus tropicalis} genome

To identify NRSE sites in the $X$. tropicalis genome, we performed an in silico screen of the genome for a $17 \mathrm{bp}$ degenerate NRSE consensus motif (NTYAGMRCCNNRG MSAG) generated from 32 bona fide REST target genes in human, rodents, and chicken $[25,26]$. The consensus NRSE motif has two highly conserved regions ( $5^{\prime}$ half and 3 ' half) separated by a linker region that consists of 2 poorly conserved nucleotides (in bold) [27]. The consensus NRSE motif does not include the lesser-conserved 4 nucleotides at the $3^{\prime}$ end found in the canonical 21 bp consensus [25]. We retrieved all NRSE sites and annotated each site based on genomic location (Additional file 1: Table S1). We found 742 NRSE sites with 340 permutations in the Xenopus genome. The consensus sequence of the Xenopus NRSE varies slightly from that of human. For example, whereas nucleotide $A$ is predominant at position 7 in the human NRSE consensus, both $A$ and $G$ are in high occupancy at this position in Xenopus (chi-square test, $\mathrm{p}<1.0 \mathrm{E}-6)$ (Figure 1A).

REST binds to NRSEs in intergenic and intragenic regions [26]. To determine the location of NRSE motifs with respect to genes, we used a cut-off distance of NRSE to genes of $100 \mathrm{~kb}$ to facilitate the comparison of our screen to one in mammals using the same degenerate sequence [25]. In human, mouse, and Xenopus, the majority of the NRSEs are located in the flanking regions of genes at a distance greater than $20 \mathrm{~kb}$ (Figure 1B). Next, we identified the number of motifs within $100 \mathrm{~kb}$ of a gene (Figure 1C). Common in both mammals and Xenopus, 80-

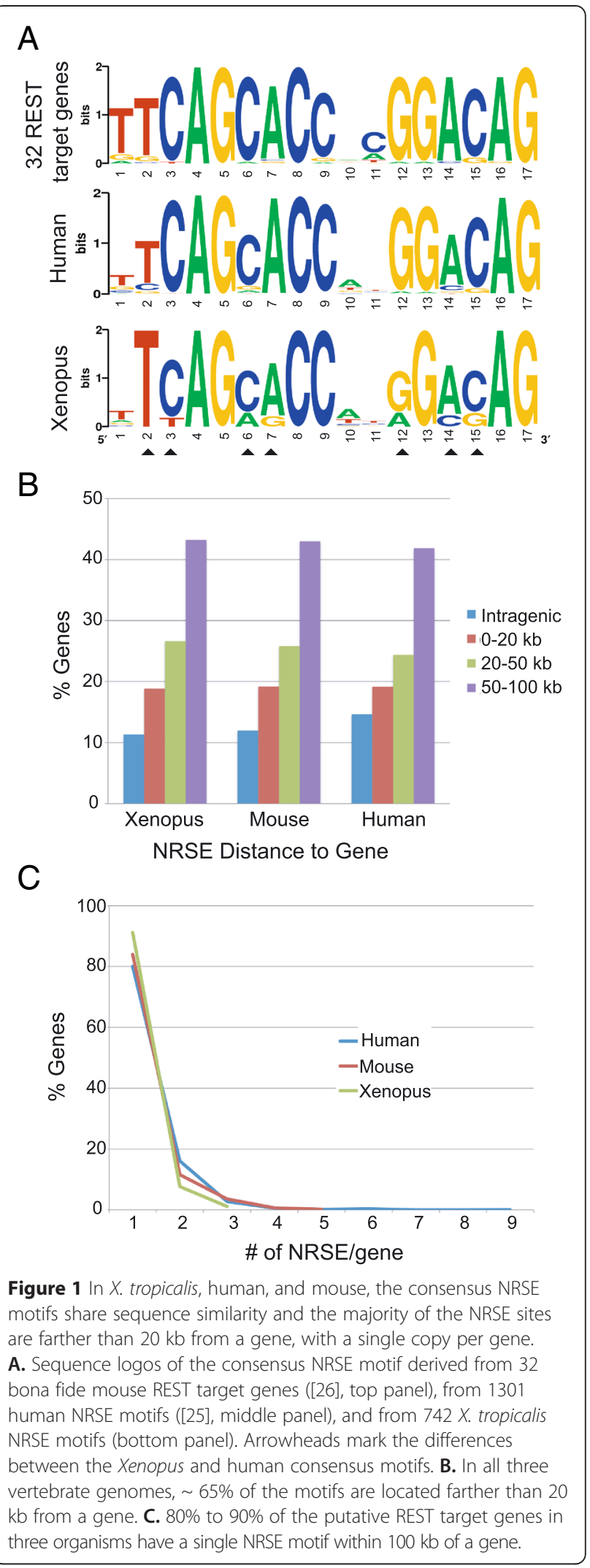


90\% of putative REST target genes are associated with a single motif; less than $10 \%$ of the targets genes are associated with two or more motifs (Figure 1C).

To determine the proportion of Xenopus NRSEs that are directly orthologous to the human NRSEs, we retrieved pairwise alignments of human (GRCh37) and $X$. tropicalis (JGI 4.2) genomes generated by genome-wide comparison using Blastz [28] from the UCSC genome browser [29] and analyzed the homologous sequences for the presence of NRSE sites. With a chain score cutoff of 5000, the summed length of homologous Xenopus regions in the pairwise alignments was $657,812,008 \mathrm{bp}$, or $2 \%$ of the Xenopus genome. We identified 85 homologous regions with sizes ranging from $42-2667$ bp that have NRSE motifs in the Xenopus homolog. However, only 12 of these 85 have an NRSE motif in the human homolog. Thus, $11.5 \%$ of the Xenopus NRSE sites are in regions of the genome with homology to the human genome, and only $14 \%$ of those regions have NRSE sites in both species. The small number of homologous regions with NRSEs is likely due to the low level of homology in non-coding regions between frogs and humans.

In total, we demonstrated that the NRSE consensus, distance from gene, and the number of motifs within $100 \mathrm{~kb}$ of a gene are similar in Xenopus, mouse, and human. However, the locations of NRSE motifs in homologous regions are not conserved among frogs and humans.

\section{Species-specific features of $X$. tropicalis NRSEs}

The Xenopus consensus motif deviates slightly from that of human and mouse. To determine where these differences lie, we first determined the frequency of each NRSE motif permutation in the genome. The degenerate NRSE sequence used to search the genome can produce 4076 permutations; however, only 340 permutations were represented in the $X$. tropicalis genome. The 340 motif permutations in 742 unique genomic loci had varying frequencies in the genome. Nearly 200 motif permutations are present only once in the genome while the most abundant motif is replicated 59 times (Figure 2A). The most common motif in the human genome is the third most common in the X. tropicalis genome. The only difference between these two motifs is a single nucleotide change in the linker region; $\mathrm{T}$ at position 11 of the Xenopus motif and $C$ in human. It has been shown that the length of the linker region, but not the identity of the nucleotides, is important for the function of REST [27]. Therefore, the differences we found in the linker region are not likely to have an effect on the binding efficiency and gene silencing capacity of REST.

To identify the Xenopus-specific motifs, we compared the Xenopus NRSEs to the human and mouse motifs. Among the 340 Xenopus NRSE motif permutations, only $70(20.5 \%)$ are in all three genomes (Figure 2B). $22.9 \%$ of

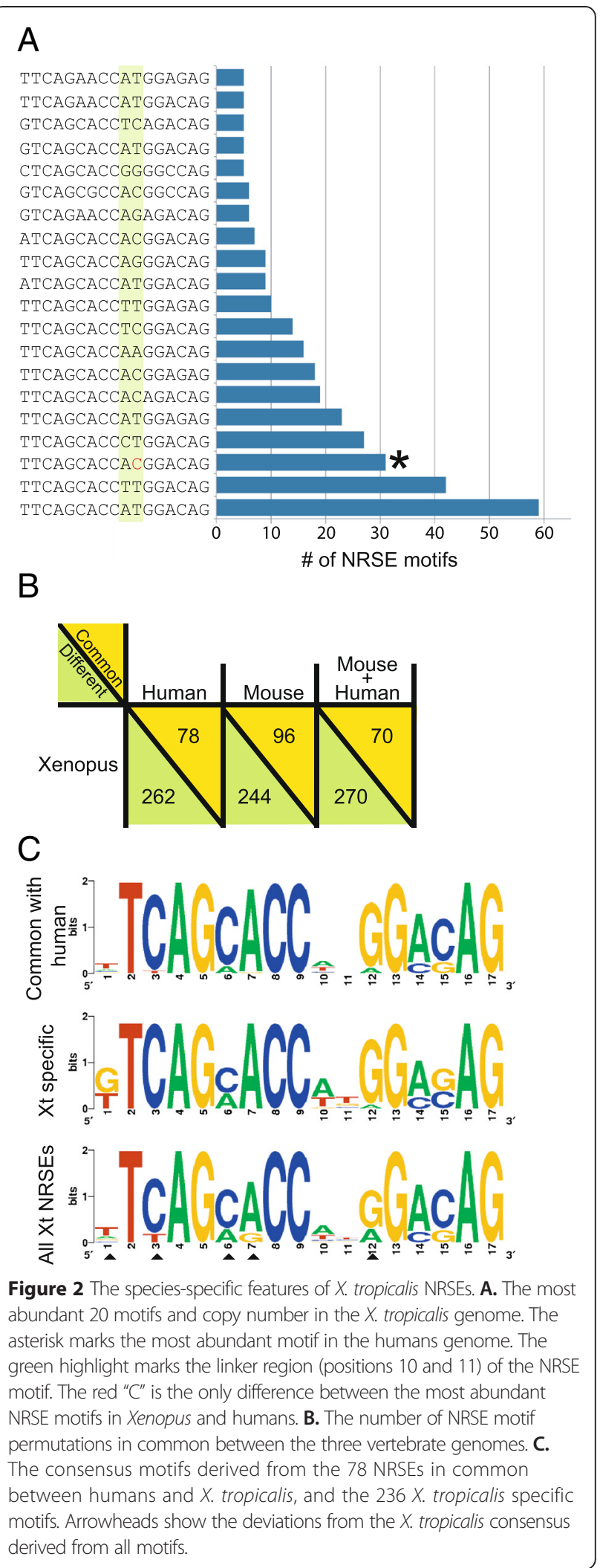

A GTCAGCACCTCAGACAG GTCAGCACCATGGACAG CTCAGCACCGGGGCCAG GTAGCGCCACGGCCAG TTCAGCACCAGGGACAG ATCAGCACCATGGACAG TCAGCACCTTGGAGAG TTCAGCACCAAGGACAG TCAGCACCACGGAGAG TTCAGCACCACAGACAC CAGCACCATGGAGAG TTCAGCACCACGGACAG TCAGCACCTTGGACA

B 
the Xenopus NRSE motifs are found in the human genome with 8 motifs exclusively shared between the two, and $28.2 \%$ of the Xenopus NRSEs are in the mouse genome with 26 motifs shared exclusively. Thus, approximately $70 \%$ (236 out of 340 motifs) of the X. tropicalis motifs are unique to the Xenopus genome (Figure 2B).

We generated a $X$. tropicalis specific consensus NRSE from 236 motifs and a Xenopus-human consensus motif from the 78 motifs shared between human and Xenopus (Figure 2C). There are significant shifts in abundance of nucleotides between Xenopus and human at positions 6 in the $5^{\prime}$-half of the motif, at position 10 in the linker region, and at position 15 in the 3 '-half (chi-square test, $\mathrm{p}<1.0 \mathrm{E}-6)$. These variations contributed to the deviation of the Xenopus consensus from the mammalian one and the generation of a species-specific version of the NRSE consensus motif.

\section{$17-24 \%$ of NRSE motifs in the human, mouse and frog} genomes are located in regions devoid of protein-coding genes

We found that $22 \%$ of all Xenopus NRSE sites are located in what we call "gene-distant regions" (GD), which are regions greater than $100 \mathrm{~kb}$ from protein-coding genes (Figure 3A). To investigate the function of the NRSEs in GD, we first compared the GD consensus motif to that of NRSEs located within $100 \mathrm{~kb}$ of a protein coding gene. There was no difference in consensus motif sequences distinguishing the motifs in low gene occupancy regions as non-functional (Additional file 2: Figure S1). To test whether these motifs may be involved in the regulation of non-coding RNAs (ncRNAs), we analyzed the relationship of the NRSEs with ncRNAs in the human genome since these are better annotated than Xenopus ncRNAs and there are fewer gaps than in the Xenopus genome. Assembly gaps increase error in estimations of distances between NRSEs and genes. Using our screen to retrieve NRSEs in the human genome, we identified 4058 motifs. We eliminated 12 of the 4058 NRSEs, because they are located on unassigned scaffolds, and therefore prone to errors in distance calculations. The distribution of distances from 4046 NRSEs to the nearest protein-coding genes ranges from 0 to $2,070,000 \mathrm{~kb}$, with a mean of $118,800 \mathrm{~kb}$ and a median of only 10,470 kb. Of the 4046 NRSEs, $980(24 \%)$ are located in GD. Of the NRSEs in gene-distant regions, 597 (61\%) are located within $100 \mathrm{~kb}$ of ncRNAs, while $2408(51 \%)$ of all NRSEs are associated with ncRNAs genome-wide. Of the ncRNA classes provided by Ensembl (lncRNA, miRNA, rRNA, snRNA, snoRNA), NRSEs are associated most frequently with lncRNAs; 485 (49\%) of the NRSEs in GD are located within $100 \mathrm{~kb}$ of lncRNA genes, while 1610 (39\%) of all NRSEs are associated with lncRNA genome-wide (Figure 3B).

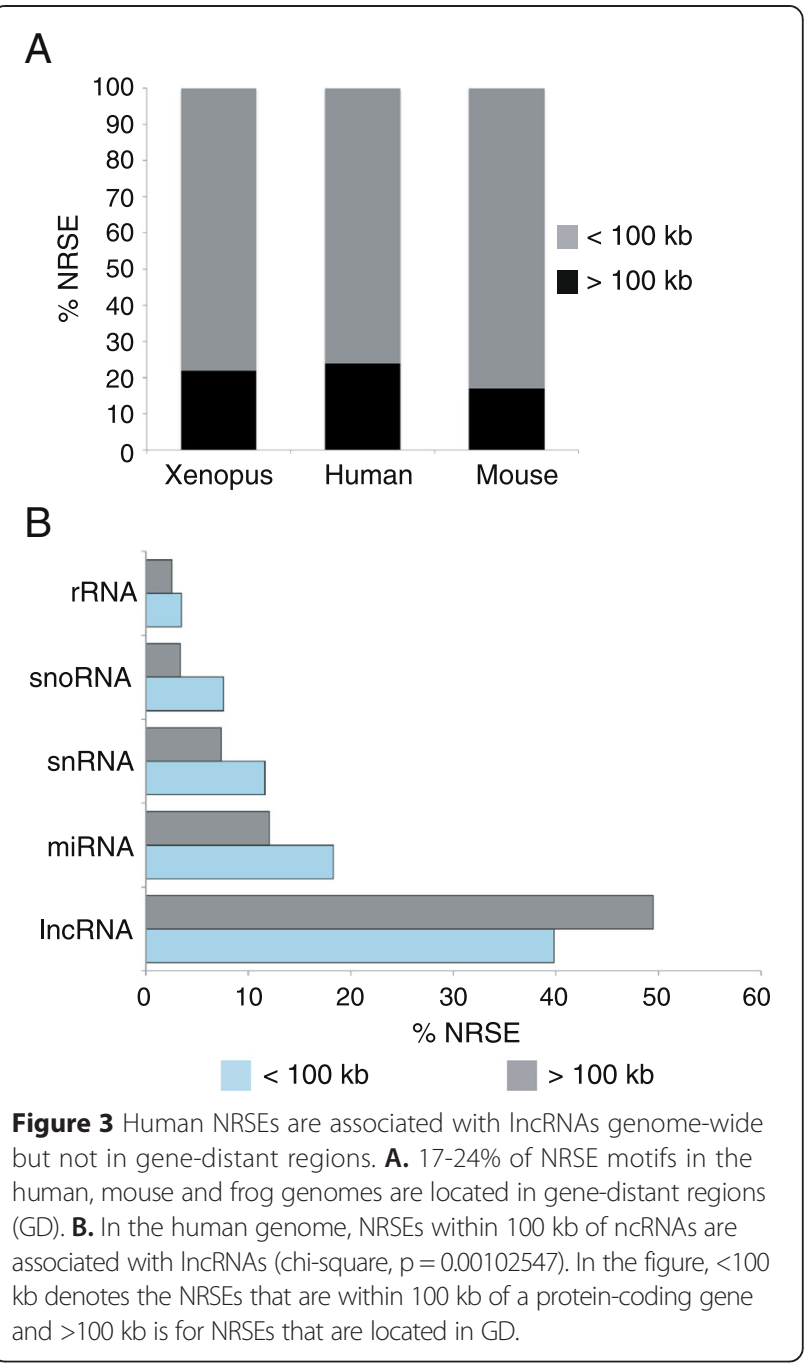

We performed chi-square tests to determine whether the numbers of NRSEs associated with ncRNA and protein-coding genes were greater than expected by chance. Expected counts were estimated by shuffling the coordinates of the human NRSE motifs. To test for significant associations of NRSE with the five ncRNA classes (lncRNA, miRNA, rRNA, snRNA, snoRNA) and protein-coding genes on a genome-wide basis, the coordinates of the 4046 NRSE were shuffled within each chromosome 1000 times such that shuffled NRSE coordinates were not allowed to fall within assembly gaps. For each class of ncRNA gene, the number of NRSEs within $100 \mathrm{~kb}$ of a gene was determined for each shuffled dataset, and the average numbers of 1000 datasets were used as expected counts in chi-square tests (Additional file 3: Table S3). We found that NRSE sites genome-wide are associated with lncRNAs more often than expected by chance (chi-square test, $\mathrm{p}=$ 0.00102), but there are no significant associations of NRSE with any other class of ncRNA. Not surprisingly, 
NRSEs are found within $100 \mathrm{~kb}$ of protein-coding genes more often than expected by chance $(\mathrm{p}=0.0008)$. The significant association of NRSEs with lncRNAs on a genome-wide basis may have been due to the frequency of IncRNAs in close proximity to protein-coding genes.

We next evaluated the subset of 980 NRSEs that are not located within $100 \mathrm{~kb}$ of protein-coding genes (i.e. within GD). Again, we shuffled the NRSE coordinates within each chromosome 1000 times. In addition to disallowing shuffled NRSE coordinates to fall within assembly gaps, we did not allow shuffled NRSEs to fall within 100 $\mathrm{kb}$ of protein-coding genes. Using the average counts from 1000 datasets to estimate numbers of NRSE within $100 \mathrm{~kb}$ of ncRNAs, the chi-square tests did not show a significant association of NRSE with any class of ncRNA given the NRSE were located in GD (Additional file 3: Table S3).

\section{A conserved group of putative REST target genes is} enriched in neuronal development and function We identified 1396 unique protein-coding genes in the $X$. tropicalis genome that are within $100 \mathrm{~kb}$ of one or more NRSE motifs (Additional file 1: Table S1).
To distinguish functional groups within the gene list, we retrieved Gene Ontology (GO) terms for X. tropicalis genes using Ensembl Biomart (Additional file 4: Table $\mathrm{S} 4$ ). The GO terms were further categorized into fourteen general functional groups (Figure 4A).

To determine whether there was a conserved core of REST target genes in vertebrates, we first identified the genes in common within $100 \mathrm{~kb}$ of the 12 conserved NRSEs in the homologous regions of the Xenopus and human genomes. We identified 22 genes within $100 \mathrm{~kb}$ of the 12 conserved NRSEs in the Xenopus genome (Additional file 5: Table S2) and 64 genes in the human genome with 9 genes in common (bdnf, dnajc5b, mtmr14, pou4f1, rnf219, setd5, slc4a1, trim55, and ubtf). Gene ontology analysis showed that of the 9 common genes, bdnf [30-32], pou4fl [33,34], rnf219 [35] and setd5 [36-38] have functions in neurogenesis and disease of the nervous system.

We broadened the search for a conserved set of vertebrate target genes to include targets shared with mouse, human and Xenopus. We first identified all of the genes within $100 \mathrm{~kb}$ of an NRSE motif in human and mouse
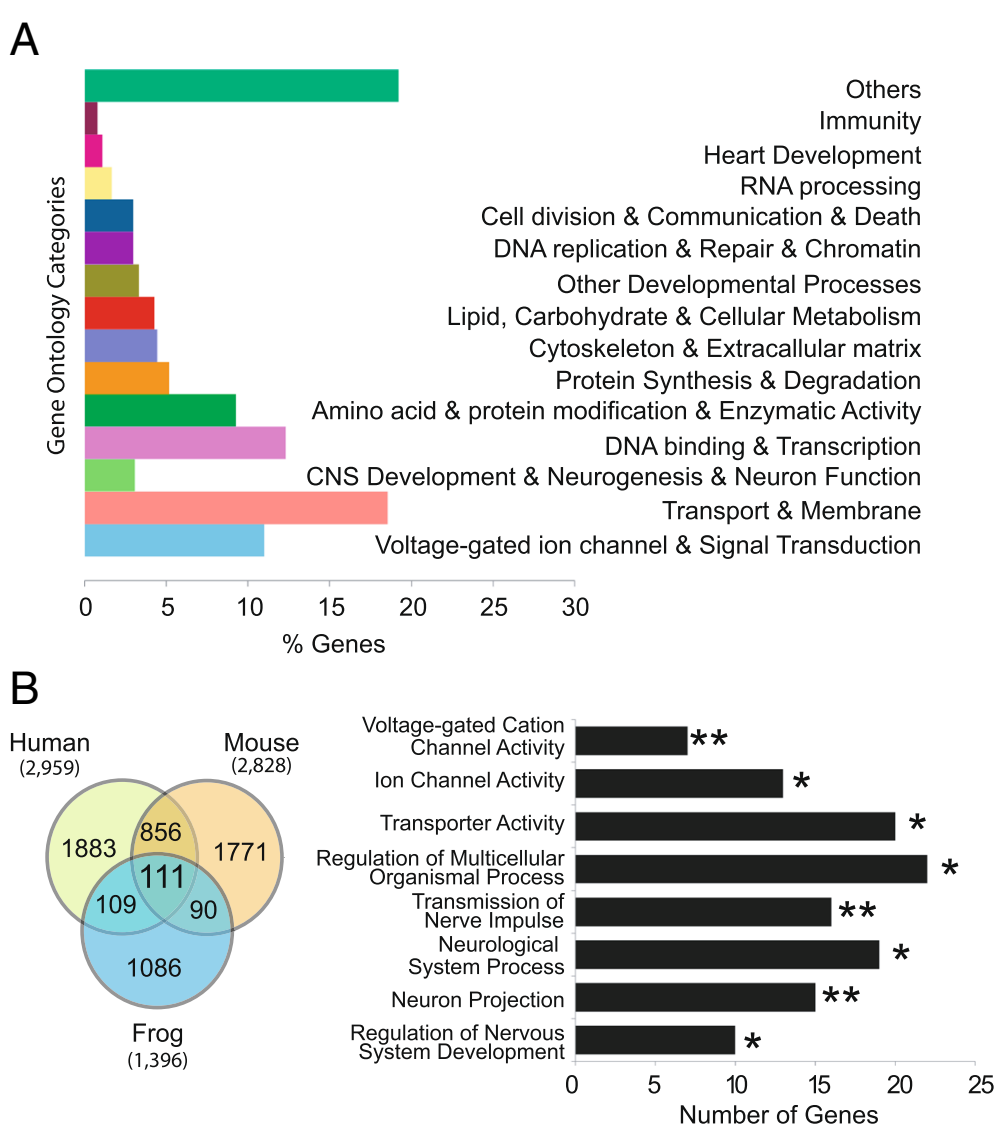

Figure 4 Gene Ontology classification of putative NRSE target genes. A. Genes were linked to 14 functional groups or "others" subgroup based on GO descriptions. B. The 111 common NRSE target genes in human, mouse, and frog genomes are enriched in neuronal functions. All categories are statistically significant with single and double asterisks showing p-values $<0.05$ and $<0.01$, respectively. 
(Figure 4B). Only $111(8 \%)$ of the $X$. tropicalis genes within $100 \mathrm{~kb}$ of an NRSE motif (with average distance of 47,954 bp and median of 46,385 bp) are also putative REST targets in mouse and human based on comparison of gene names (Additional file 6: Table S5). The list of shared genes, categorized into functional GO groups using g: Profiler software (Table 1), are significantly enriched in neuronal functions, including voltage-gated cation channel activity, transmission of nerve pulse, neuron projection, and regulation of nervous system development (Figure 4B). These genes may comprise a core group of protein-coding genes that are central to REST-meditated gene silencing.

\section{In silico validation of the NRSE screen}

The Xenopus NRSE screen successfully identified 25 of 32 bona fide REST target genes [26]. We also used two in silico approaches to validate the screen. First, we searched Xenbase, a publicly available gene expression database for $X$. tropicalis and X. laevis [39], for the spatial expression profile of each gene with an NRSE motif as determined by whole mount in situ hybridizations. Neuronal- and heart-restricted genes [40] including those expressed in the neural plate, neural tube, brain, spinal cord, and eyes (but not neural progenitors or stem cells) were considered true positives. Although REST splice variants are also expressed in thymus, kidney, testis, lung, spleen, and muscle [13], the experimental evidence for REST function in these peripheral tissues is lacking. Therefore, we did not include gene expression in such peripheral tissues to indicate regulation by REST. Expression elsewhere was considered a false positive. Out of 1396 genes, only 206 had in situ expression data in Xenbase (Table 2). Among 206 genes, expression of 141 genes $(68 \%)$ is restricted to neuronal and heart tissues. Of these 141 genes, 16 are among the 111 putative REST target genes common in human, mouse, and frog genomes.

Table 1 Gene Ontology classification of conserved core 111 common genes using g:profiler

\begin{tabular}{llll}
\hline GO term definition & GO term & No. of genes & P-value \\
\hline $\begin{array}{l}\text { Regulation of Nervous System } \\
\text { Development }\end{array}$ & GO:0051960 & 10 & $4.32 \mathrm{E}-02$ \\
$\begin{array}{l}\text { Regulation of Multicellular } \\
\text { Organismal Process }\end{array}$ & $\mathrm{GO}: 0035637$ & 15 & $5.52 \mathrm{E}-04$ \\
Neurological System Process & $\mathrm{GO}: 0050877$ & 19 & $1.63 \mathrm{E}-02$ \\
Transmission of Nerve Impulse & $\mathrm{GO} 00019226$ & 16 & $8.55 \mathrm{E}-04$ \\
Neuron Projection & $\mathrm{GO} 0043005$ & 22 & $2.95 \mathrm{E}-02$ \\
Transporter Activity & $\mathrm{GO}: 0005215$ & 20 & $1.01 \mathrm{E}-02$ \\
lon Channel Activity & $\mathrm{GO} 00005216$ & 13 & $3.29 \mathrm{E}-04$ \\
Voltage-gated Cation Channel & $\mathrm{GO} 0022843$ & 7 & $2.96 \mathrm{E}-02$ \\
Activity & & & \\
\hline
\end{tabular}

The published REST Chip-seq data in human [41-43] was also used to validate the putative REST targets genes conserved among Xenopus, mouse and human in our in silico screen. The REST Chip-seq analysis identified 57 (51\%) of the 111 putative REST target genes shared between mouse, human and frog indicating these as true targets of REST (Table 2). Thus, the in silico validation of our screen suggests that we successfully identified putative REST target genes in the Xenopus genome.

\section{In vivo validation of the NRSE screen}

The link between REST and protein degradation has not been well established although both have fundamental roles in neurogenesis [44]. Towards understanding the regulatory relationship between REST and protein degradation, we studied 4 F-box genes $f b x o 16, f b x o 41, f b x l 7$, and $f b x l 10$, identified in our screen. F-box proteins are the E3 ligase components of RING type ubiquitin ligases [45]. The NRSE motifs associated with the F-box genes are located upstream or downstream of the genes at a distance $>50 \mathrm{~kb}$ except for Fbxl10, which has an NRSE within $2.3 \mathrm{~kb}$ of the gene start (Figure $5 \mathrm{~A}$ ). There is an intervening gene between $f b x o 16, f b x o 41$, and $f b x l 7$ and the NRSE. To determine if these genes are restricted to neuronal tissues, we analyzed their expression in $X$. tropicalis embryos using in situ hybridization. All four genes are expressed in the developing embryo from gastrula to tailbud stages (Figure 5B). In early gastrulae (st 10.5 ), the genes are weakly expressed in the ectoderm with greater expression in the dorsal neuroectoderm. However, expression increases at the neurula stage (st. 17) and all genes are primarily expressed in the neural tube. Whereas $f b x o 16, f b x o 41$, and $f b x l 7$ are pan neural, $f b x l 10$ is localized to the anterior-most and posteriormost regions of the neural plate. At early tailbud stages (st. 25 \& st. 30), all genes are expressed in the brain with $f b x o 41, f b x l 7$ and $f b x l 10$ also expressed in the eyes and branchial arches. In Xenopus, REST is maternal and uniformly expressed in the ectoderm during gastrula stages (Additional file 7: Figure S2). However, the expression is diffuse in the neurula embryo including the neural folds and then later restricted to the brain and spinal cord in tailbud stages (Additional file 7: Figure S2 and [2]). At the cellular level, REST is expressed in neural progenitors and stem cells but excluded from differentiating and mature neurons $[9,13]$. Our expression analysis confirmed the NRSE screen and showed that the expression of four putative F-box genes is localized to neural tissues during Xenopus development.

\section{Discussion}

With a genome-wide screen, we identified 742 NRSE motifs associated with 1396 protein-coding genes (within $100 \mathrm{~kb}$ ) in the $X$. tropicalis genome. We compared the 
Table 2 In silico validation of the Xenopus NRSE screen with in situ hybridization and REST CHIP-seq. Putative REST target genes were categorized into neuronal/heart or non-neuronal/non-heart expression based on in situ hybridization data collected from Xenbase

\begin{tabular}{|c|c|c|}
\hline in situ hybridization & & REST CHIP-Seq \\
\hline Neuronal/Heart Expression 141 genes & Non-neuronal/non-heart Expression & 57 genes \\
\hline $\begin{array}{l}\text { Bsx, arl6ip1, cox5a, ag1, cacna1h, adarb1, ahctf1, } \\
\text { asb8, churc1, cpsf2, cpeb1, dact1, adprh, bmp7.1, } \\
\text { bsn, cldn5, asap1, arid4a, cdca8, cdk1, calu, } \\
\text { colec11, arl8a, bri3, admp, cdh12, arx, celf2, } \\
\text { cdc45, cacna1a, col18a1, cACNA1C, calb1, } \\
\text { clasp1, copg, crb2, dlx4, dmrta2, dnal4, dvl1, } \\
\text { egr1, eif4h, elavl4, elk1, ephb1, erbb4, ern2, } \\
\text { esr10, esyt2, ext1, fam54b, fgf12, frzb2, fzd3, } \\
\text { gabbr2, gabra3, gabra5, gbx2.1, gbx2.2, gcat, }\end{array}$ & $\begin{array}{l}\text { Alb, alg3, amy2b, anxa4, arf1, armc4, baiap2l1, } \\
\text { bsg, col1a1, CREB3L2, ctsc, cxcr7, dazap1, dcdc2, } \\
\text { fgf14, fuz, gamt, gfpt1, gorasp2, grhl1, gstp1, } \\
\text { igsf9b, impdh1, iqgap1, iqgap2, itga8, klf5, } \\
\text { krt5.7, laptm4a, Idlrap1, mmp9, mst1r, myos, } \\
\text { nbn, ndufaf3, nodal, nom1, odc1, pcdh8, } \\
\text { ppp1r3c.1, rab18, rab8a, rnd1, sept2, sept9, } \\
\text { sfrp5, sgk2, snd1, sox17b.1, sox2, trappc2, } \\
\text { tspan7, tspan8, ttll4, upk1b, ventx1.2, ventx2.1, }\end{array}$ & $\begin{array}{l}\text { Angptl6, ap1s1, ap3b2, brsk2, bsx, cacna1a, } \\
\text { cacna1h, cacna2d2, cdh22, cdb4, chd5, chat, } \\
\text { cpsf3, cyp27b1, decr1, ebf1, fgf14, glra1, grin1, } \\
\text { hes3, icmt, kcnc3, kcnh4, kndc1, Ihx3, Ihx5, } \\
\text { lin37, march11, nefm, neto1, nr2f1, nup133, } \\
\text { ogdh, olfm3, pafah1b1, pcgf6, pipox, plbd2, } \\
\text { plbd2, pou4f1, ptk2b, pusl1, qsox2, rdh8, ric8b, } \\
\text { rnf219, sdsl, slc35f4, slc4a1, slc4a1ap, slc5a11, } \\
\text { syt4, taf5, vwc2l, xkr7, zcchc14, rasgrf1 }\end{array}$ \\
\hline
\end{tabular}
hnrnpa1, hpcal1, id2 igfbp4, insm1, ism1, kaz, klf11, klhdc4, Ihx2, Ihx3, Ihx5, limk1, mef2d, $\mathrm{mnt}$, mnx1, myl7, myo1c, myo1d, nbl1, ncoa5, nefm, neurod1, neurog3, nol10, nr2f2, pcdh10, pcmtd1, pla2g7, plxnb1, pnhd, pou4f1, prph, rab34, rab7a, rasip1, rax, rbm38, rgs20, rhbdd3, ric8a, rps3, scn2a, scn3a, selt, sema3a, siah2, slc32a1, slc3a2, slit1, smad4, smarca4, snai1, sox14, sphk1, spry2, srsf5, srsf6, supt6h, suv420h1, tbx5, tcea1, tmub2, tpm4, tubgcp4, vamp1, vav2, wdr5, wdr73, wdr74, wnt16, wnt3a, wnt9b

Out of 1,396 genes, 206 had expression data, 141 (68\%) of which had expression in neuronal/heart tissues. REST CHIP-Seq targets were retrieved from [41-43]. The bold emphasis indicates the 16 genes among the 111 putative REST target genes common in human, mouse, and frog genomes.

data of our screen in Xenopus to that of screens in human and mouse [25]. The three vertebrate data sets have similar features in terms of the number of NRSE motifs associated with genes, their distance to genes, and the GO classification of genes with NRSEs. Furthermore, we identified four F-box ubiquitin ligases as putative REST target genes in Xenopus with expression restricted to the neuronal tissues in developing embryos. Our analysis, therefore, identified a new regulatory relationship between REST and the components of protein degradation machinery (Figure 6).

Screening the Xenopus genome, we identified the majority of the previously identified bona fide human REST target genes including NaV1.2 [26], one of the first REST target genes identified. However, we failed to identify SCG10 (also known as STMN2), another well-studied REST target gene [1]. In fact, no NRSE motif was present

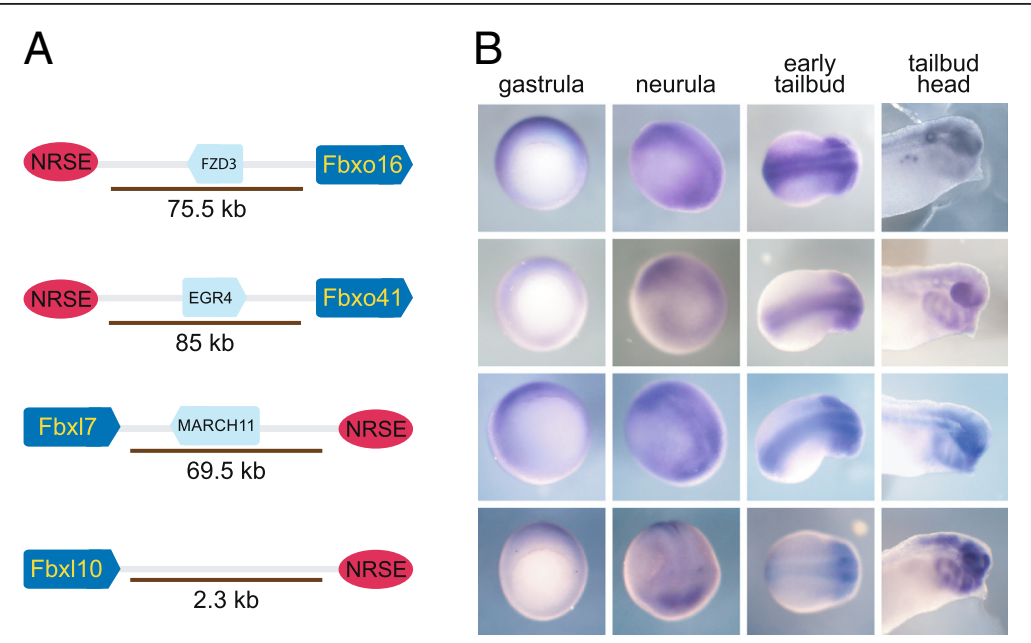

Figure $\mathbf{5}$ In vivo validation of the Xenopus NRSE screen. Four F-box ubiquitin ligases identified in the NRSE screen are expressed in the neuronal tissues of $X$. tropicalis. A. The genomic localization of the genes with respect to NRSE motifs and $\mathbf{B}$. their mRNA expression hybridization during $X$. tropicalis development. Gastrula embryos are ventral view with dorsal to the top. Neurula embryos are dorso-lateral view with anterior to the right. Early tailbud embryos are dorsal view with anterior to the right. Tailbud heads are lateral view with anterior to the right. The arrows point the direction of genes. Intervening genes are in turquoise. Cartoons are not to scale. 


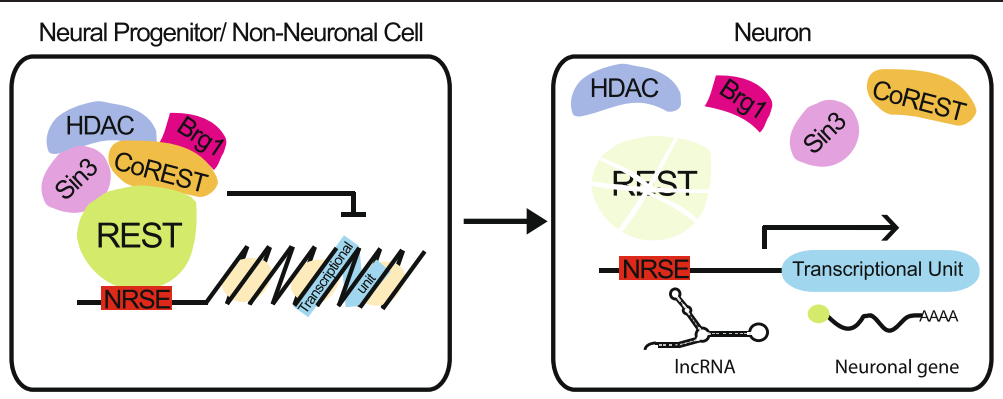

Figure 6 REST mediated repression of coding and non-coding gene expression. In neural progenitors and non-neuronal cells, REST facilitates silencing of expression by binding to highly conserved NRSE elements and recruiting co-repressors and chromatin remodeling agent to convert the topology of the local DNA to heterochromatin. During neurogenesis, REST transcription is down regulated and the protein is degraded. The absence of REST in neuronal cells allows the expression of neuron specific protein-coding genes and non-coding RNAs including micro and long non-coding RNAs.

on the scaffold containing SCG10. To investigate the possibility that SCG10 is regulated by an unconventional NRSE motif, we searched the scaffold for partial (i.e. 5'half or $3^{\prime}$-half) and bipartite NRSE motifs (i.e. the NRSE motifs with $5^{\prime}$ and $3^{\prime}$ halves separated by more than 2 linker nucleotides) [27]. We identified $175^{\prime}$-half and 262 3 '-half motifs within $100 \mathrm{~kb}$ of SCG10 but no bipartite motifs. This suggests that the regulation of SCG10 expression in Xenopus either involves an unconventional regulatory mechanism such as REST binding to partial motifs or is regulated independent of REST.

Our genome-wide analysis was based on a conserved consensus NRSE motif that allowed degeneracy at certain positions in the motif. However, it did not identify non-traditional NRSE motifs such as bipartite, partial or species-specific motifs, which could only be identified through Chip-seq analysis. However, our analysis using the conserved NRSE motif did identify the conserved target genes among vertebrates.

It is surprising that only $8 \%$ of NRSE target genes are conserved among the three vertebrate genomes. This could be due to the fact that REST is able to bind degenerate NRSE motifs. In fact, the NRSE motifs in functionally validated REST target genes show differences in sequence but yet satisfy REST binding [26]. The flexibility in binding may allow the emergence of new sites through nucleotide substitution, and hence, the recognition of new genes. As expected, we identified a larger number of conserved NRSE target genes among mammals than across the greater evolutionary distance of the three vertebrates. We found more than $30 \%$ of NRSE target genes to be conserved between human and mouse but only $8 \%$ were conserved among in human, mouse, and frog (Figure 4B). The more conserved genes might have undergone high selective constraint against changes in the REST sites, and are functionally essential. Lesserconserved genes with relaxed constraints may have allowed the emergence of new NRSE sites.
While our analysis did not reveal a significant association of NRSEs with lncRNA in gene-distant regions (GD), we did find NRSEs to be associated with lncRNA genomewide. Our analysis could not exclude the possibility that the significant association of NRSEs with IncRNA was due to the close proximity of IncRNA and protein-coding genes. However, evidence for the regulation of lncRNAs by REST continues to emerge [21-24].

\section{Conclusions}

With a screen for REST binding sites in the X. tropicalis genome and a comparison of the characteristics of these binding sites in mouse, human and frog, we determined that NRSEs are most commonly located greater than 20 $\mathrm{kb}$ from a protein-coding gene in single copies, that there is a Xenopus-specific consensus, that only $20.5 \%$ of the Xenopus NRSEs are also in mouse and human and that there is a conserved core of putative REST target genes enriched in neuronal function in these three vertebrates. We also identified four F-box proteins as putative target proteins thereby linking ubiquitin-mediated degradation with regulation of neurogenesis.

\section{Methods}

In silico detection and characterization of NRSE binding sites

A genome-wide search for NRSE binding sites in the $X$. tropicalis genome (genome assembly version JGI 4.2 [46]) was performed as described [25]. A unique identifier was given to each NRSE motifs based on the genomic localization. To facilitate the comparison to those screens in mammalians [25], similar criteria and settings were used in analysis, including the same consensus NRSE sequence, with human genome assembly GRCh37 [47] and mouse genome assembly MGSCv37 [48]. Motif logos were generated using WebLogo (http://weblogo. berkeley.edu/logo.cgi) [49]. 


\section{Identification of homologous regions in the human genome}

To identify regions in the human chromosome that were homologous to Xenopus regions containing NRSE motifs, pairwise genome alignments between human genome assembly GRCh37 and X. tropicalis genome assembly JGI4.2 were downloaded from the UCSC Genome Browser web site (http://hgdownload.soe.ucsc.edu/goldenPath/xenTro3/ vsHg19/xenTro3.hg19.all.chain.gz). We performed our NRSE screen on sequences provided in the chain file to determine whether regions in the Xenopus genome with NRSE motifs had homologous regions in the human genome with NRSE motifs.

\section{Annotation of genes within $100 \mathrm{~kb}$ of NRSE}

Xenopus, human, and mouse annotations for both coding- and non-coding gene sets were retrieved from Ensembl which uses GENCODE, a merge of the automatic annotation from Ensembl and the manually curated annotation from Havana. $X$. tropicalis genes with descriptions, genomic location, and Gene Ontology (GO) terms were downloaded from Ensembl Biomart [50]. The genes common in the human, mouse, and frog NRSE screens were identified through the comparison of gene names.

A higher ordering of GO terms was achieved by manual assignment to 14 functional groups (Voltage-gated ion channel and signal transduction, Transport and membrane, CNS development, neurogenesis and neuron function, DNA binding and transcription, Amino acid, protein modification and enzymatic activity, Protein synthesis and degradation, Cytoskeleton and extracellular matrix, Lipid, carbohydate and cellular metabolism, DNA replication, repair and chromatin, Cell division, communication and death, RNA processing, Heart development, Other developmental processes, Immunity). For the common genes in the human, mouse, and frog screens, GO classification was done with g: Profiler (http://biit.cs.ut.ee/gprofiler/) [51] using default settings.

\section{Analysis of NRSE association with ncRNA}

We used the human genome assembly GRCh37 and Ensembl human annotation release GRCh37.75 to investigate potential associations of NRSE with ncRNA. The annotation file in gtf format was retrieved from the Ensembl ftp site (ftp.ensembl.org). We parsed the gtfformatted file into separate files for protein-coding genes, IncRNA, miRNA, rRNA, snoRNA and siRNA, keeping only the gene features so that genes with multiple transcripts would not be counted more than once. We used the intersectBed program from the BedTools package [52] to identify motifs that fell within or outside the ranges of interest. In order to identify motifs located within or further than $100 \mathrm{~kb}$ of a gene, we modified gtf files to extend gene coordinates $100 \mathrm{~kb}$ in each direction. For example, to identify motifs further than $100 \mathrm{~kb}$ from protein-coding genes (i.e. gene-distant regions or GD), we used intersectBed with the $-\mathrm{v}$ option (show features that do not overlap) to output NRSE motif coordinates that do not overlap protein-coding gene coordinates which had been extended by $100 \mathrm{~kb}$. We then used intersectBed with the $-\mathrm{u}$ option (list each feature in set A once if it overlaps set B) to intersect the coordinates of the previous output with lncRNA coordinates which had been extended $100 \mathrm{~kb}$, to identify NRSE that were greater than $100 \mathrm{~kb}$ from protein-coding genes but within $100 \mathrm{~kb}$ of the IncRNA. We repeated this for each ncRNA class, and we performed similar intersections with the entire NRSE dataset to investigate genomewide associations.

For chi-square tests to determine whether the numbers of NRSE within $100 \mathrm{~kb}$ of ncRNA and protein-coding genes were greater than expected by chance, we estimated expected counts by shuffling the coordinates of the human NRSE motifs using the shuffle program from the BedTools suite. First, we used the Table Browser available from UCSC Genome Browser to create a file of genome assembly gaps, and used it with the -excl option of the shuffle program to exclude gaps from possible shuffled locations. The -chrom option was used so that locations were permuted within each chromosome instead of randomly in the genome. Shuffling was performed 1000 times each on the total human NRSE dataset (4046 motifs with chromosome coordinates) and the dataset of 980 NRSE in GD, so that we could perform separate chi-square tests for each dataset. For each dataset, 1000 shuffled NRSE gtf files were used as input to intersectBed with the $-\mathrm{u}$ option to perform intersections with coordinates of different ncRNA classes that had been extended by $100 \mathrm{~kb}$ in each direction. In the case of the gene desert dataset, shuffled motif locations were excluded from within $100 \mathrm{~kb}$ of proteincoding genes (i.e. they had to remain in GD). This resulted in 1000 genome-wide and 1000 gene desert counts for NRSE located within $100 \mathrm{~kb}$ of each class of ncRNA. We used the average of 1000 counts as the expected count for each chi-square test.

\section{Whole mount in situ hybridization}

Whole-mount in situ hybridization (WISH) was performed as described [53,54] with the following modifications: pre-hybridization treatment was extended to overnight and an additional 1X SSC wash (15 min, room temperature) was added. $X$. tropicalis embryos were gifts from M. Khokha (Yale U., Connecticut). X. tropicalis Fbxl7, Fbxl10, Fbxo16, and Fbxo41 clones were gifts from R. Harland (U. of California, Berkley). The riboprobes were digoxigenin-labeled. 


\section{Ethics statement}

This study was carried out in strict accordance with the recommendations in the NRC Guide for the Care and Use of Laboratory Animals. The protocol was approved by the Georgetown University Animal Care and Use Committee (GUACUC, Protocol: 13-016). Euthanasia was performed under the American veterinary medical association guidelines, and all efforts were made to minimize suffering.

\section{Availability of supporting data}

The data sets supporting the results of this article are included within the article and its additional files.

\section{Additional files}

Additional file 1: Table S1. $X$. tropicalis protein-coding genes within $100 \mathrm{~kb}$ of one or more NRSE motif.

Additional file 2: Figure S1. Consensus sequence logos for NRSE motifs associated with protein-coding genes and in gene-distant regions are similar. NRSE motifs associated with protein-coding genes were located $\leq 100 \mathrm{~kb}$ distance. The NRSEs in GD were located $>100 \mathrm{~kb}$ from a protein-coding gene

Additional file 3: Table S3. Chi-square tests for association of NRSES with ncRNAs in the entire human genome and in gene-distant regions based in shuffling NRSE coordinates.

Additional file 4: Table S4. $G O$ terms for $X$. tropicalis protein-coding genes within $100 \mathrm{~kb}$ of an NRSE motif.

Additional file 5: Table S2. The genes associated with 12 NRSE motifs identified in homologous regions of human and $X$. tropicalis genomes.

Additional file 6: Table S5. REST target genes conserved in mouse, human and frog.

Additional file 7: Figure S2. The expression of REST during Xenopus laevis development using in situ hyridization. In the egg, animal pole is to the top. Gastrula embryos are ventral view with dorsal to the top. Tailbud embryo is lateral view with anterior to the left.

\section{Abbreviations}

REST: Repressor element 1 silencing transcription factor; NRSE: Neuronrestrictive silencing element; ncRNA: Non-coding RNA; IncRNA: Long-non coding RNA; miRNA: MicroRNA; rRNA: Ribosomal RNA; snRNA: Small nuclear RNA; snoRNA: Small nucleolar RNA; MeCP: Methyl-CpG-binding protein; BTRC: Beta-transducin repeat containing protein; Fbxw: F-box protein with WD40 domain; Fbxl: F-box protein with leucine rich repeat; Fbxo: F-box protein with domain other than WD40 or leucine rich repeat; BAF53a: Brg/ Brm associated factor 53a; hESC: Human embryonic stem cell; GD: Gene-distant regions; GO: Gene ontology; WISH: Whole-mount in situ hybridization.

\section{Competing interests}

The authors declare that they have no competing interests.

\section{Authors' contributions}

CC wrote the initial PERL script to identify NRSE sites in the $X$. tropicalis genome. BSY analyzed the data and compared it to that of mouse and human, and performed in situ hybridizations. CE revised the manuscript and performed the chi-square tests for statistical analysis of REST association with ncRNAs. ES participated in the design and coordination of the study and helped to draft the manuscript. All authors read and approved of the final manuscript.

\section{Acknowledgements}

The authors thank M. Khokha for X. tropicalis embryos, R. Harland for F-box clones and Ximiao He for revising the original PERL program. This study was funded in part by NIH HD060698 to ES.

\section{Author details}

'Department of Biology, Georgetown University, 411 Regents Hall, Washington, DC 20057, USA. ${ }^{2}$ Division of Animal Sciences, University of Missouri, Columbia, MO 65211, USA. ${ }^{3}$ Division of Plant Sciences, University of Missouri, Columbia, MO 65211, USA.

Received: 15 May 2014 Accepted: 28 April 2015

Published online: 14 May 2015

\section{References}

1. Chen ZF, Paquette AJ, Anderson DJ. NRSF/REST is required in vivo for repression of multiple neuronal target genes during embryogenesis. Nat Genet. 1998;20:136-42.

2. Armisén R, Fuentes R, Olguín P, Cabrejos ME, Kukuljan M. Repressor element-1 silencing transcription/neuron-restrictive silencer factor is required for neural sodium channel expression during development of Xenopus. J Neurosci. 2002:22:8347-51.

3. Thiel $G$, Lietz M, Cramer M. Biological activity and modular structure of RE-1silencing transcription factor (REST), a repressor of neuronal genes. J Biol Chem. 1998;273:26891-9.

4. Chong J, Tapia-Ramírez J, Kim S, Toledo-Aral J, Zheng Y, Boutros M, et al. REST: a mammalian silencer protein that restricts sodium channel gene expression to neurons. Cell. 1995:80:949-57.

5. Andrés ME, Burger C, Peral-Rubio MJ, Battaglioli E, Anderson ME, Grimes J, et al. COREST: a functional corepressor required for regulation of neural-specific gene expression. Proc Natl Acad Sci U S A. 1999;96:9873-8.

6. Grimes J, Nielsen S, Battaglioli E, Miska E, Speh J, Berry D, et al. The Co-repressor mSin3A Is a Functional Component of the REST-CoREST Repressor Complex. J Biol Chem. 2000;275:9461-7.

7. Roopra A, Sharling L, Wood I. Transcriptional Repression by Neuron-Restrictive Silencer Factor Is Mediated via the Sin3-Histone Deacetylase Complex. Mol Cell Biol. 2000;20:2147-57.

8. Yu HB, Johnson R, Kunarso G, Stanton LW. Coassembly of REST and its cofactors at sites of gene repression in embryonic stem cells. Genome Res. 2011;21:1284-93.

9. Ballas N, Grunseich C, Lu DD, Speh JC, Mandel G. REST and its corepressors mediate plasticity of neuronal gene chromatin throughout neurogenesis. Cell. 2005;121:645-57.

10. Shi Y, Lan F, Matson C, Mulligan P, Whetstine JR, Cole PA, et al. Histone demethylation mediated by the nuclear amine oxidase homolog LSD1. Cell. 2004:119:941-53.

11. Battaglioli E, Andrés ME, Rose DW, Chenoweth JG, Rosenfeld MG, Anderson $M E$, et al. Rest repression of neuronal genes requires components of the hSWI · SNF complex. J Biol Chem. 2002;277:41038-45.

12. Gao Z, Ure K, Ding P, Nashaat M, Yuan L, Ma J, et al. The master negative regulator REST/NRSF controls adult neurogenesis by restraining the neurogenic program in quiescent stem cells. J Neurosci. 2011;31:9772-86.

13. Palm K, Belluardo N, Metsis M, Timmusk T. Neuronal expression of zinc finger transcription factor REST/NRSF/XBR gene. J Neurosci. 1998;18:1280-96.

14. Mandel G, Fiondella CG, Covey MV, Lu DD, Loturco JJ, Ballas N. Repressor element 1 silencing transcription factor (REST) controls radial migration and temporal neuronal specification during neocortical development. Proc Nat Acad Sci U S A. 2011;108:16789-94.

15. Covey M, Streb J, Spektor R, Ballas N. REST regulates the pool size of the different neural lineages by restricting the generation of neurons and oligodendrocytes from neural stem/progenitor cells. Development. 2012:139:2878-90.

16. Nishimura E, Sasaki K, Maruyama K, Tsukada T, Yamaguchi K. Decrease in neuron-restrictive silencer factor (NRSF) mRNA levels during differentiation of cultured neuroblastoma cells. Neurosci Lett. 1996;211:101-4.

17. Westbrook TF, Hu G, Ang XL, Mulligan P, Pavlova NN, Liang A, et al. SCFbeta-TRCP controls oncogenic transformation and neural differentiation through REST degradation. Nature. 2008:452:370-4.

18. Wu J, Xie X. Comparative sequence analysis reveals an intricate network among REST CREB and miRNA in mediating neuronal gene expression. Genome Biol. 2006;7:R85 
19. Kuwabara T, Hsieh J, Nakashima K, Taira K, Gage FH. A small modulatory dsRNA specifies the fate of adult neural stem cells. Cell. 2004;116:779-93.

20. Yoo AS, Staahl BT, Chen L, Crabtree GR. MicroRNA-mediated switching of chromatin-remodelling complexes in neural development. Nature. 2009;460:642-6.

21. Johnson $\mathrm{R}$, Teh $\mathrm{CH}-\mathrm{L}$, Jia $H$, Vanisri RR, Pandey $T$, Lu Z-H, et al. Regulation of neural macroRNAs by the transcriptional repressor REST. RNA. 2009;15:85-96.

22. Ng SY, Bogu GK, Soh B, Stanton LW. The long noncoding RNA RMST interacts with SOX2 to regulate neurogenesis. Mol Cell. 2013;51:349-59.

23. 23. Ng SY, Johnson R, Stanton LW: Human long non-coding RNAs promote pluripotency and neuronal differentiation by association with chromatin modifiers and transcription factors. The EMBO Journal 2011;31(3):522-533.

24. Tsai M-C, Manor O, Wan Y, Mosammaparast N, Wang JK, Lan F, et al. Long noncoding RNA as modular scaffold of histone modification complexes. Science. 2010;329:689-93.

25. Bruce AW, Donaldson IJ, Wood IC, Yerbury SA, Sadowski MI, Chapman M, et al. Genome-wide analysis of repressor element 1 silencing transcription factor/neuron-restrictive silencing factor (REST/NRSF) target genes. Proc Natl Acad Sci U S A. 2004:101:10458-63.

26. Schoenherr C, Paquette A, Anderson D. Identification of potential target genes for the neuron-restrictive silencer factor. Proc Natl Acad Sci U S A. 1996;93:9881-6.

27. Patel PD, Bochar DA, Turner DL, Meng F, Mueller HM, Pontrello CG. Regulation of tryptophan hydroxylase-2 gene expression by a bipartite RE-1 silencer of transcription/neuron restrictive silencing factor (REST/NRSF) binding motif. J Biol Chem. 2007:282:26717-24.

28. Schwartz S, Kent WJ, Smit A, Zhang Z, Baertsch R, Hardison RC, et al. Human-mouse alignments with BLASTZ. Genome Res. 2003;13:103-7.

29. Rosenbloom KR, Armstrong J, Barber G, Casper J, Clawson H, Diekhans M, et al. The UCSC Genome Browser database: 2015 update. Nucleic Acids Res. 2015;43(Database issue):D670-81.

30. Conner JM, Lauterborn JC, Yan Q, Gall CM, Varon S. Distribution of brainderived neurotrophic factor (BDNF) protein and mRNA in the normal adult rat CNS: evidence for anterograde axonal transport. J Neurosci. 1997;17:2295-313.

31. Rossi C, Angelucci A, Costantin L, Braschi C, Mazzantini M, Babbini F, et al. Brain-derived neurotrophic factor (BDNF) is required for the enhancement of hippocampal neurogenesis following environmental enrichment. Eur J Neurosci. 2006;24:1850-6

32. Suliman S, Hemmings SMJ, Seedat S. Brain-Derived Neurotrophic Factor (BDNF) protein levels in anxiety disorders: systematic review and metaregression analysis. Front Integr Neurosci. 2013;7(July):55.

33. Deng M, Yang H, Xie X, Liang G, Gan L. Comparative expression analysis of POU4F1, POU4F2 and ISL1 in developing mouse cochleovestibular ganglion neurons. Gene Expr Patterns. 2014;15:31-7.

34. Zou M, Li S, Klein W, Xiang M. Brn3a/Pou4f1 regulates dorsal root ganglion sensory neuron specification and axonal projection into the spinal cord. Dev Biol. 2012;364:114-27.

35. Rhinn H, Fujita R, Qiang L, Cheng R, Lee JH, Abeliovich A. Integrative genomics identifies APOE $\varepsilon 4$ effectors in Alzheimer's disease. Nature. 2013;500:45-50.

36. Kuechler A, Zink AM, Wieland T, Lüdecke H-J, Cremer K, Salviati L, Magini P, Najafi K, Zweier C, Czeschik JC, Aretz S, Endele S, Tamburrino F, Pinato C, Clementi M, Gundlach J, Maylahn C, Mazzanti L, Wohlleber E, Schwarzmayr T, Kariminejad R, Schlessinger A, Wieczorek D, Strom TM, Novarino G, Engels $\mathrm{H}$ : Loss-of-function variants of SETD5 cause intellectual disability and the core phenotype of microdeletion 3p25.3 syndrome. Eur J Hum Genet 2014. http://www.nature.com/ejhg/journal/vaop/ncurrent/full/ejhg2014165a.html.

37. Grozeva D, Carss K, Spasic-Boskovic O, Parker MJ, Archer H, Firth HV, et al. De novo loss-of-function mutations in SETD5, encoding a methyltransferase in a 3p25 microdeletion syndrome critical region, cause intellectual disability. Am J Hum Genet. 2014:94:618-24.

38. Pinto D, Delaby E, Merico D, Barbosa M, Merikangas A, Klei L, et al. Convergence of genes and cellular pathways dysregulated in autism spectrum disorders. Am J Hum Genet. 2014;94:677-94.

39. Bowes J, Snyder K, Segerdell E, Jarabek C, Azam K, Zorn A, et al. Xenbase: gene expression and improved integration. Nucleic Acids Res. 2009;38(Database Issue):D607-12.

40. Kuwahara K, Saito $Y$, Ogawa E, Takahashi N, Nakagawa $Y$, Naruse $Y$, et al. The neuron-restrictive silencer element-neuron-restrictive silencer factor system regulates basal and endothelin 1-inducible atrial natriuretic peptide gene expression in ventricular myocytes. Mol Cell Biol. 2001;21:2085.
41. Satoh J, Kawana N, Yamamoto Y. ChIP-Seq Data Mining: Remarkable Differences in NRSF/REST Target Genes between Human ESC and ESC-Derived Neurons. Bioinform Biol Insights. 2013;7:347-55.

42. Lu T, Aron L, Zullo J, Pan Y, Kim H, Chen Y, et al. REST and stress resistance in ageing and Alzheimer's disease. Nature. 2014;507:448-54

43. Johnson R, Richter N, Bogu GK, Bhinge A, Teng SW, Choo SH, Andrieux LO, de Benedictis C, Jauch R, Stanton LW: A genome-wide screen for genetic variants that modify the recruitment of REST to its target genes. PLOS Genet 2012;8(4):e1002624

44. Saritas-Yildirim B, Silva EM. The role of targeted protein degradation in early neural development. Genesis. 2014:52:287-99.

45. Willems AR, Schwab M, Tyers M. A hitchhiker's guide to the cullin ubiquitin ligases: SCF and its kin. Biochim Biophys Acta. 2004;1695:133-70.

46. Hellsten U, Harland RM, Gilchrist MJ, Hendrix D, Jurka J, Kapitonov V, et al. The genome of the Western clawed frog Xenopus tropicalis. Science. 2010;328:633-6.

47. Church DM, Schneider VA, Graves T, Auger K, Cunningham F, Bouk N, Chen HC, Agarwala R, McLaren WM, Ritchie GRS, Albracht D, Kremitzki M, Rock S, Kotkiewicz H, Kremitzki C, Wollam A, Trani L, Fulton L, Fulton R, Matthews L, Whitehead S, Chow W, Torrance J, Dunn M, Harden G, Threadgold G, Wood J, Collins J, Heath P, Griffiths G, et al.: Modernizing reference genome assemblies. PLoS Biol 2011;9(7):e1001091.

48. Church DM, Goodstadt L, Hillier LW, Zody MC, Goldstein S, She X, Bult CJ, Agarwala R, Cherry JL, DiCuccio M, Hlavina W, Kapustin Y, Meric P, Maglott D, Birtle Z, Marques AC, Graves T, Zhou S, Teague B, Potamousis K, Churas C, Place M, Herschleb J, Runnheim R, Forrest D, Amos-Landgraf J, Schwartz DC, Cheng Z, Lindblad-Toh K, Eichler EE, et al.: Lineage-specific biology revealed by a finished genome assembly of the mouse. PLoS Biol 2009:7(5):e1000112.

49. Crooks GE, Hon G, Chandonia J-M, Brenner SE. WebLogo: A Sequence Logo Generator. Genome Res. 2004;14:1188-90.

50. Flicek P, Amode MR, Barrell D, Beal K, Brent S, Carvalho-Silva D, et al. Ensembl 2012. Nucleic Acids Res. 2012;40(Database issue):D84-90.

51. Reimand J, Kull M, Peterson H, Hansen J, Vilo J. g:Profiler-a web-based toolset for functional profiling of gene lists from large-scale experiments. Nucleic Acids Res. 2007:35(Web Server issue):W193-200.

52. Quinlan A. BEDTools: The Swiss-Army Tool for Genome Feature Analysis. Curr Protoc Bioinforma. 2014:47:11.12.1-11.12.34

53. Harland RM. Appendix G: In Situ Hybridization: An Improved Whole-Mount Method for Xenopus Embryos. Methods Cell Biol. 1991;36:685-95.

54. Hemmati-Brivanlou A, Frank D, Bolce ME, Brown BD, Sive HL, Harland RM Localization of specific mRNAs in Xenopus embryos by whole-mount in situ hybridization. Development. 1990;110:325-30.

\section{Submit your next manuscript to BioMed Central and take full advantage of:}

- Convenient online submission

- Thorough peer review

- No space constraints or color figure charges

- Immediate publication on acceptance

- Inclusion in PubMed, CAS, Scopus and Google Scholar

- Research which is freely available for redistribution 